\title{
HST \& VLA Imaging of the Symbiotic Star HM Sge
}

\author{
S. P. S. Eyres, M. F. Bode \\ Astrophysics Research Institute, Liverpool John Moores' University, \\ Twelve Quays House, Egerton Wharf, Birkenhead, CH41 1LD, UK
}

\begin{abstract}
Symbiotic stars are a heterogenous group of interacting binaries, where the combination of a mass-losing giant and a hot compact companion create conditions ideal for studying interacting winds, dust formation, nebular shaping and accretion phenomena. We present $H S T$ and VLA observations of the symbiotic nova HM Sge as an example of our work. In this case, we characterise hot and cool parts of the extended nebula, determine the angular binary separation to be $40 \pm 9$ milli-arcsec, and the interstellar reddening to be no more than $\mathrm{E}(\mathrm{B}-\mathrm{V})=0.35$. Comparison with a suggested binary separation of 50 au gives a distance of $1250 \pm 280 \mathrm{pc}$, consistent with the sparsely-sampled distance-extinction mapping in the direction of HM Sge. A number of other observations of additional high spatial-resolution observations of symbiotic stars have been completed or are being planned.
\end{abstract}

\section{Introduction}

Symbiotic stars are interacting binaries, in which a mass-losing giant or Miratype star (cool component or CC) orbits with a hot, compact star (hot component or HC) usually thought to be a white dwarf (see Kenyon 1986; Mikołajewska 1997). The result is a giant wind from the CC ionised by the $\mathrm{HC}$ radiation field. Many symbiotic stars have an active $\mathrm{HC}$, in which either frequent brightening and even flickering is seen, or occasional nova-like outbursts lasting years to decades, instead of the months to years typical of classical novae. This activity appears to be accompanied by a hot, fast wind from the HC. This range of conditions means that symbiotic stars exhibit dust formation, wind and binary interaction, nebular shaping and accretion phenomena.

We are pursuing a programme of high-spatial resolution imaging, intially with radio interferometers (Eyres et al. 1996; Dougherty et al. 1996; Richards et al. 1999; Watson et al. 2000), and recently also with HST WFPC2 imaging in seven filters (Eyres et al. 2000, submitted; other in preparation). As an example of our work, we present here some of our results for HM Sge. This is a symbiotic nova, which brightened by $\sim 6$ magnitudes in $U B V$ in 1975 and has remained around $\mathrm{V}=11.5$ ever since (Mürset \& Nussbaumer 1994). The outbursting HC has been modelled as a contracting pseudo-photosphere, with the most recent estimates from IUE observations giving $T_{\text {eff }}=200000 \mathrm{~K}$ and $R_{H C}=0.083 \mathrm{R}_{\odot}$ (Mürset, Wolff \& Jordan 1997). The star is classified as D-type, meaning the IR spectrum is dominated by a dust continuum. Our MERLIN observations correlated the structure in the inner nebula at $6 \mathrm{~cm}$ with that seen with the $H S T$ in the UV (Eyres et al. 1996), while further observations showed that the structure of the inner nebula changed over a few years (Richards et al. 1999). An interacting wind model lead Richards et al. (1999) to suggest that 
these changes are coupled to the binary motion with $\mathrm{P}_{\text {orb }}=80 \mathrm{yrs}$, and that the binary is currently at maximum elongation on the sky. They suggested a binary separation of 50 au.

\section{Results}

Some of our $H S T$ images are presented in Fig. 1. These are representative of the structures seen in all the HST WFPC2 filters observed (F218W, F437N, F469N, F487N, F502N, F547M, F656N), and in the VLA images. To save space, we describe the radio results without the aid of a figure. Features $\mathrm{N}, \mathrm{C}$ and $\mathrm{S}$ in Fig. 1(a) are visible to some extent in all the HST WFPC2 images, and in the $23 \mathrm{GHz}$ VLA image, where the shape is almost identical to that seen in the F502N filter. The $8.56 \mathrm{GHz}$ image is of significantly lower resolution, but a central feature clearly due to a merging of $\mathrm{N}, \mathrm{C}$ and $\mathrm{S}$ is apparent, and there is an extension to the SW coincident with feature 1.

The images in Figs. 1(a) and (b) are dominated by [O III] lines at $\sim 5000 \AA$ and $4363 \AA$ respectively. The ratio of these lines is used as nebular temperature and density diagnostics (see Osterbrock 1989). Fig 1(d) shows the result of taking the ratio of Fig. 1(a) and Fig. 1(b). There is clearly a structure of high line ratio $(>15)$ in the $\mathrm{SW}$, coincident with feature 1 . There are also enhancements in the ratio at features 2,3 and 4, and the extension of feature $\mathrm{N}$ to the west of the central inner nebula. The SW wedge is coincident with regions with radio brightness temperatures between $140 \mathrm{~K}$ and $2200 \mathrm{~K}$ at $8.56 \mathrm{GHz}$, which allows us to conclude that the SW wedge is cooler than the remaining extended nebula, although the exact temperatures depend on the accuracy of the $H S T$ absolute flux scale and the fraction of the extended nebula resolved-out by the VLA.

Using the images from the HST WFPC2 F218W and F547M filters (which we take to sample the UV and red continuum respectively), we have used Gaussian fits to the PSF to determine the positions of the HC and the CC directly. Estimation of the expected contributions of the HC and the CC in the F218W and F547M filter images shows that the HC dominates in the former, while the $\mathrm{CC}$ dominates in the latter. We find an angular binary separation of $40 \pm 9$ milliarcsec, and a position angle (north through east) for the line between the two stars (the "binary axis") of $130^{\circ} \pm 10^{\circ}$. The direct determination of the binary positions is a break-through in symbiotic star research, which will have important consequences for interacting wind models.

We have used the HST WFPC2 image in the F487N filter [Fig. 1(c)], which is dominated by the $\mathrm{H} \beta$ line, and the VLA $23 \mathrm{GHz}$ image to derive an extinction map (see Osterbrock 1989 for a discussion of this technique). This traces the dust distribution in the inner arcsec of the nebula, showing it to be clumpy. We take the minimum value of $\mathrm{E}(\mathrm{B}-\mathrm{V})=0.35$ (equivalent to $A_{V} \simeq 1.1$ ) in this extinction map to be the best estimate of the true interstellar extinction.

\section{Discussion}

Our angular binary separation compared with the value of 50 au predicted by Richards et al. (1999) would give a distance of $1250 \pm 280 \mathrm{pc}$, if the binary is currently at or close to maximum elongation as suggested by those authors. This is consistent with the result given in Ivison et al. (1991), by a different method.

Spectro-polarimetry by Schmid et al. (2000) found a prefered angle of $\sim 123^{\circ}$, and they concluded that this was the current orientation of the binary axis. This agrees with our binary axis position angle of $130^{\circ} \pm 10^{\circ}$, but contra- 


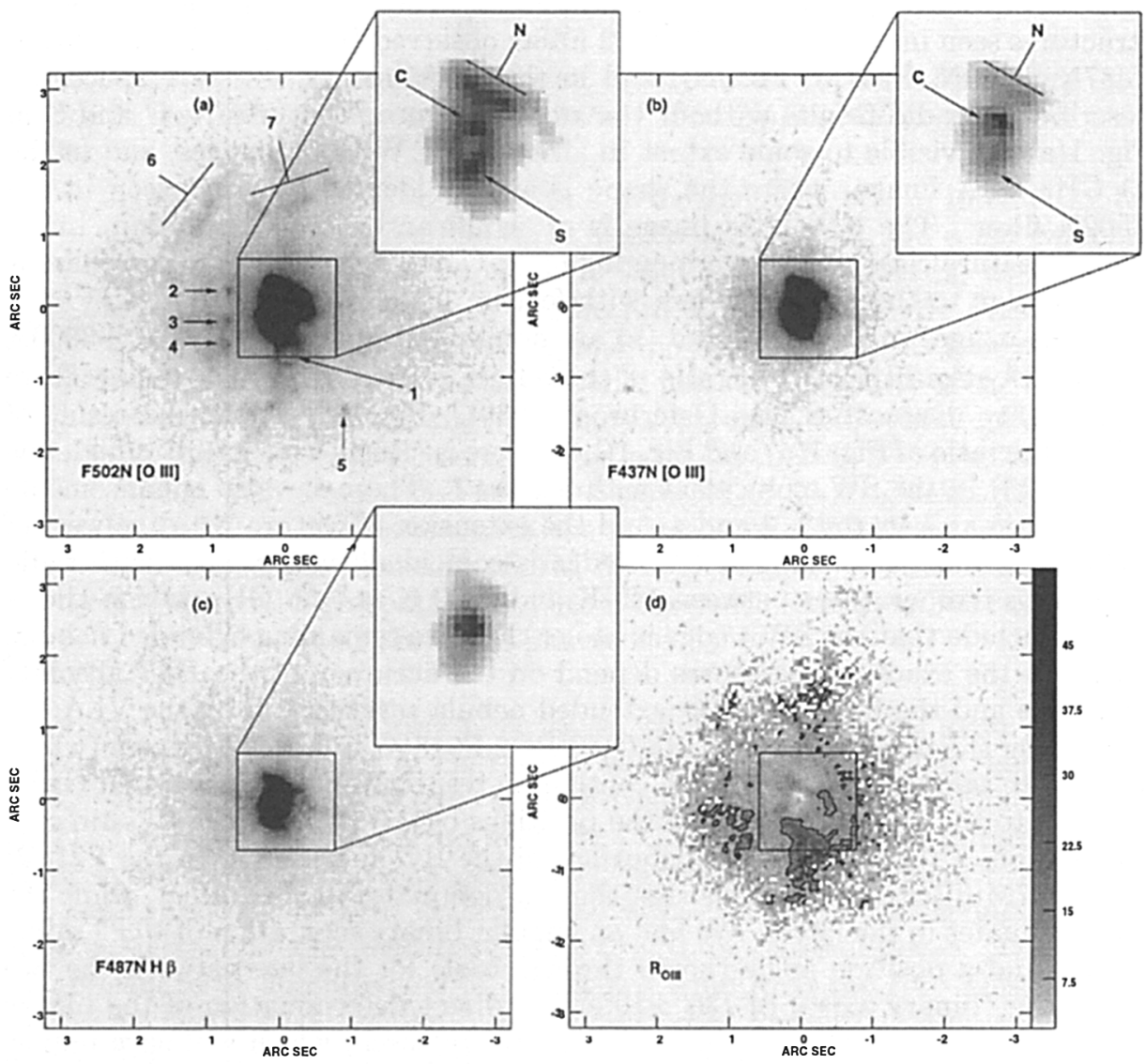

Figure 1. HST images of HM Sge, dereddenned for $\mathrm{E}(\mathrm{B}-\mathrm{V})=$ 0.35 , in the WFPC2 filters (a) F502N; greyscale range $9.1 \times 10^{-15}$ to $1.3 \times 10^{-12}$ (main panel) and $1.3 \times 10^{-12}$ to $2.9 \times 10^{-11}$ (inset); (b) $\mathrm{F} 437 \mathrm{~N}$; greyscale range $4.7 \times 10^{-15}$ to $2.3 \times 10^{-13}$ (main panel) and $2.3 \times 10^{-13}$ to $10^{-11}$ (inset); and (c) $\mathrm{F} 487 \mathrm{~N}$; greyscale range $3.8 \times 10^{-15}$ to $9.6 \times 10^{-13}$ (main panel) and $9.6 \times 10^{-13}$ to $2.5 \times 10^{-11}$ (inset). Units are erg s ${ }^{-1} \mathrm{~cm}^{-2} \AA^{-1} \operatorname{arcsec}^{-2}$. Image (d) is the ratio of images (a) and (b) with greyscale range 2 to 50 , and a single contour at a line ratio of 15 . Uncertainties in the ratio are of order $10 \%$. 
dicts the suggestion by Richards et al. (1999) that the CC is directly north of the HC.

The extinction towards HM Sge appears to be variable, with values as high as $A_{V}=13.5$ (Whitelock 1988, Munari \& Whitelock 1989), and as low as $A_{V}=$ 1.64 (Ivison et al. 1991). All these values include a circumstellar contribution, and we believe that our value $\mathrm{E}(\mathrm{B}-\mathrm{V})=0.35$ or $A_{V} \simeq 1.1$ is the most robust estimate of the true interstellar extinction to date. The clumpy nature of the dust in the inner nebula suggested by our extinction mapping is consistent with the variations in $A_{V}$ being due to periodic dust obscuration events, and possibly dust production, in the inner nebula (as suggested by e.g. Thronson \& Harvey 1981).

It should be emphasised that our use of the [O III] line ratios and the radio brightness maps together for nebular diagnostics is a new application of this technique, providing spatially-resolved temperature and density information for the first time in symbiotic stars. We suggest that the cool SW wedge is associated with the $\mathrm{CC}$ wind, the shape of which has been modified by interaction with the $\mathrm{HC}$ radiation field and fast wind. The form of feature 1 is supportive of this conclusion. Alternatively, the cool SW wedge may be a result of shadowing of the outer nebula by feature $\mathrm{S}$, which our extinction mapping shows to have the greatest concentration of dust in the inner nebula.

We acknowledge the contribution of Prof. A. R. Taylor, Dr. R. J. Davis and Mr. M. M. Crocker to this work.

\section{References}

Dougherty, S. M., Bode, M. F., Lloyd, H. M., Davis, R. J., Eyres, S. P. S., 1995, MNRAS, 272, 843

Eyres, S. P. S., Kenny, H. T., Cohen, R. J., Lloyd, H. M., Dougherty, S. M., Davis, R. J. \& Bode, M. F. 1995, MNRAS, 274317

Ivison, R. J., Bode, M. F., Roberts, J. A., Meaburn, J., Davis, R. J., Nelson, R. F., Spencer, R. E. 1991, MNRAS, 249, 374

Kenyon, S. J. 1986, The symbiotic stars (Cambridge: CUP)

Mikołajewska, J. 1997, Physical processes in symbiotic binaries and related systems (Warszawa: Fundacja Astronomii Polskiej im. Mikołaja Kopernika)

Munari, U. \& Whitelock, P. A. 1989, MNRAS, 237, 45P

Mürset, U. \& Nussbaumer, H., 1994, A\&A, 282, 586

Mürset, U., Wolff, B., Jordan, S., 1997, A\&A, 319, 201

Osterbrock, D. E., 1989, Astrophysics of Gaseous Nebulae and Active Galactic Nuclei (Mill Valley, CA: University Science Books)

Richards, A. M. S., Bode, M. F., Eyres, S. P. S., Kenny, H. T., Davis, R. J. and Watson, S. K. 1999, MNRAS, 305, 380

Schmid, H. M., Corradi, R., Krautter, J., Schild, H. 2000, A\&A, 355, 261

Thronson, H. A. and Harvey, P. M. 1981, ApJ, 248, 584

Watson, S. K., Davis, R. J., Bode, M. F., Eyres, S. P. S., 2000, MNRAS, 311, 449

Whitelock, P. A. 1988, in IAU Colloq. No. 103, The symbiotic phenomenon, eds. Mikołajewska, J., Friedjung, M., Kenyon, S. J. \& Viotti, R. (Dordrecht: Kluwer), 47 